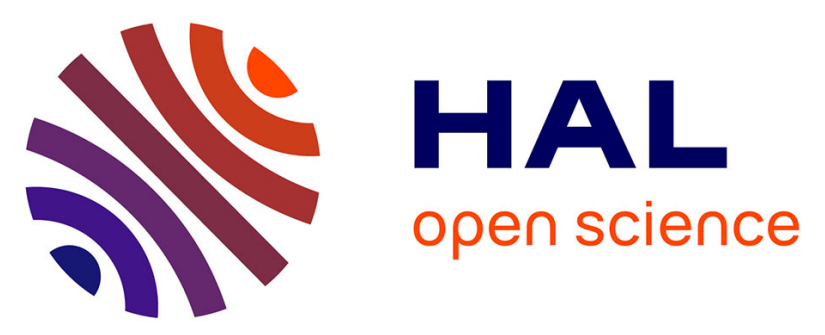

\title{
La ligne à grande vitesse Est-européenne en région Champagne-Ardenne: un outil au service d'un processus de métropolisation?
}

Sylvie Bazin, Christophe Beckerich, Marie Delaplace, Sophie Masson

\section{- To cite this version:}

Sylvie Bazin, Christophe Beckerich, Marie Delaplace, Sophie Masson. La ligne à grande vitesse Esteuropéenne en région Champagne-Ardenne: un outil au service d'un processus de métropolisation?. RTS - Recherche Transports Sécurité, 2006. hal-02077973

\section{HAL Id: hal-02077973 \\ https://hal.univ-reims.fr/hal-02077973}

Submitted on 16 Apr 2019

HAL is a multi-disciplinary open access archive for the deposit and dissemination of scientific research documents, whether they are published or not. The documents may come from teaching and research institutions in France or abroad, or from public or private research centers.
L'archive ouverte pluridisciplinaire HAL, est destinée au dépôt et à la diffusion de documents scientifiques de niveau recherche, publiés ou non, émanant des établissements d'enseignement et de recherche français ou étrangers, des laboratoires publics ou privés. 


\title{
La ligne à grande vitesse est-européenne en région Champagne-Ardenne Un outil au service d'un processus de métropolisation?
}

\section{The East European high-speed line in the Champagne-Ardenne region Does it serve the metropolization process?}

\author{
Sylvie Bazin ${ }^{\text {a }}$, Christophe Beckerich ${ }^{\text {a }}$, Marie Delaplace ${ }^{\mathrm{a}, *}$, Sophie Masson ${ }^{\mathrm{b}}$ \\ Adresses professionnelles des auteurs à la date d'acceptation de l'article \\ a Université de Reims Champagne-Ardenne, Laboratoire CERAS-OMI, 57 bis rue Pierre Taittinger, 51096 Reims cedex \\ ${ }^{\mathrm{b}}$ Université de Perpignan, Via Domitia, Laboratoire GEREM, 52 avenue de Villeneuve, 66860 Perpignan cedex
}

Reçu le 17 octobre 2005 ; accepté le 20 juillet 2006

\section{Résumé}

La métropole rassemble des fonctions diversifiées, notamment des fonctions tertiaires supérieures, rayonne sur un environnement régional, national et international et fonctionne en réseau avec les autres grandes villes et les villes moyennes qui l'entourent. Si, selon cette définition, il n'existe pas de réelle métropole régionale en Champagne-Ardenne, l'entrée en service de la ligne à grande vitesse esteuropéenne prévue en juin 2007 est néanmoins susceptible de modifier cette situation, non seulement en renforçant l'accessibilité de Reims à Paris, ainsi qu'à l'ensemble des métropoles françaises, mais également en créant une forme de coordination entre les différents acteurs locaux.

\section{Abstract}

A metropolis brings together varied functions, in particular higher tertiary-sector functions, exerts an influence at a regional, national and international level, and functions in networks with the other large cities and medium-sized cities around it. While, according to this definition, there is no real metropolis in the Champagne-Ardenne region, the future East European high-speed line is likely to modify this situation not only by improving accessibility of Rheims from Paris and all the other French metropolises, but also by creating a degree of coordination between the various local actors.

Mots clés : Ligne à grande vitesse ; Métropolisation ; Coordination ; Champagne-Ardenne

\footnotetext{
* Auteur correspondant.

Courriel : marie.delaplace@univ-reims.fr

(ㄷ) 2006 Lavoisier SAS et INRETS. Tous droits réservés.
} 


\section{Introduction}

La DATAR définit une métropole comme une très grande ville, à la fois par la taille de sa population et de celle de l'agglomération qu'elle anime, par son poids économique, politique, social et culturel et par son pouvoir d'attraction et de diffusion (DATAR, 2003). La métropole rassemble des fonctions diversifiées, notamment des fonctions tertiaires supérieures, rayonne sur un environnement régional, national et international et fonctionne en réseau avec les autres grandes villes et les villes moyennes qui l'entourent.

En générant une diminution de la durée du trajet entre certaines agglomérations de la région Champagne-Ardenne et Paris, la ligne à grande vitesse est-européenne rapprochera la principale agglomération desservie en Champagne-Ardenne, l'agglomération rémoise, de Paris et de sa banlieue, ainsi que de l'Est (Strasbourg, Allemagne, Suisse). Par le biais de l'interconnexion, elle la rapprochera également de certaines villes de province reliées au réseau à grande vitesse, notamment Lille, Nantes, Rennes et Bordeaux. L'objectif est ici d'évaluer dans quelle mesure cette ligne à grande vitesse, en améliorant l'accessibilité de l'agglomération rémoise, peut conforter l'émergence d'un processus de métropolisation ${ }^{1}$. Les acteurs politiques et institutionnels rémois comptent effectivement sur cette meilleure accessibilité pour renforcer l'attractivité de la ville (faible aujourd'hui, comparativement à d'autres agglomérations) et anticipent un processus de métropolisation associé à cette attractivité. Après avoir mis en évidence le caractère encore embryonnaire de ce processus, nous montrons que l'arrivée de la grande vitesse est susceptible d'influer, sous certaines conditions, sur certains critères de la métropolisation, notamment sur celui de la coordination entre les acteurs locaux.

\section{Le processus de métropolisation autour de l'agglomération rémoise}

Si le processus de métropolisation repose sur des attributs spécifiques, force est de constater que l'ensemble de ces attributs n'est pas présent dans l'agglomération rémoise.

\subsection{Un processus de métropolisation reposant sur des attributs spécifiques}

Il n'y a pas de consensus, ni de définition clairement énoncée et acceptée de façon universelle de la notion de

\footnotetext{
${ }^{1}$ Cet article s'appuie sur un contrat de recherche commandité par le Conseil régional de Champagne-Ardenne, qui consiste en une analyse prospective des effets de la ligne à grande vitesse est-européenne en région Champagne-Ardenne. Les propos n'engagent néanmoins que ses auteurs. Le rapport final est consultable sur le site du Conseil régional ChampagneArdenne (www.cr-champagne-ardenne.fr) à la rubrique Territoire et Environnement, puis Transport.
}

métropole et de celle de métropolisation. Ainsi, de la métropole d'équilibre 2 à la métropole polynucléaire ${ }^{3}$ en passant par la métapole ${ }^{4}$, le foisonnement terminologique qualifiant les métropoles renvoie à des réalités de configurations et d'agglomérations urbaines d'activités et de populations aussi complexes que radicalement opposées. Selon PH. Derycke $(1999 ; 1)$, malgré d'innombrables travaux, en France et à l'étranger, l'accord est loin d'être unanime parmi les économistes sur ces deux termes, sur les implications des processus qui les sous-tendent et sur le contenu précis des concepts qui prétendent les abstraire ou les résumer. Certaines villes sont implicitement reconnues comme étant dotées d'attributs métropolitains, tandis que d'autres usent de cette terminologie sans véritable référence claire leur permettant de valider ce statut ${ }^{5}$. Le concept de métropolisation est également ambivalent, même s'il existe un consensus scientifique pour considérer la métropolisation comme un ensemble de processus dynamiques qui transforment une ville en métropole (Derycke, 1999 ; 2). Si cette définition reste encore à construire, de nombreuses tentatives d'éclaircissement (voir par exemple (Corade, 1994)) aboutissent néanmoins à un certain consensus autour des attributs métropolitains. Nous retiendrons la définition proposée par G. Wackermann (2000 ; 65) : La vraie métropole est un pôle urbain majeur doté de la totalité ou de la quasitotalité des fonctions urbaines au niveau qualitatif le plus élevé [...] Elle concentre des activités de commandement dans les domaines les plus divers, participe à la maîtrise de l'espace mondial et appuie son rayonnement sur un territoire continu relativement vaste. Les attributs métropolitains seraient les suivants.

- Des attributs logistiques : la métropole est un point nodal majeur. Elle génère des flux, y compris immatériels, et assure également la concentration et la redistribution (polarisation) de flux divers (hommes, biens, informations, capitaux) et

\footnotetext{
2 Politique mise en place en France dans les années 1960, devant permettre un rééquilibrage de la répartition des activités, jusque-là concentrées en Île de France, vers de grandes villes.

${ }^{3}$ Constituée de réseaux de villes à plusieurs pôles relativement équilibrés, contrairement aux réseaux macrocéphaliques dans lesquels la métropole écrase le réseau par son poids (comme Paris ou Buenos Aires à l'échelle nationale, ou encore Toulouse à l'échelle régionale). Université et diversité des villes dans le monde, www.Keepschool.com, 2006.

${ }^{4}$ François Ascher la définit comme un ensemble d'espaces dont tout ou partie des habitants, des activités économiques ou des territoires sont intégrés dans le fonctionnement quotidien (ordinaire) d'une métropole. Les espaces qui la composent sont profondément hétérogènes et pas nécessairement contigus.[...] Elles sont mono ou polynucléaires, plus ou moins agglomérées ou éclatées, hétérogènes, polarisées ou segmentées (Ascher, 1995).

${ }^{5}$ Le terme métropole est souvent indifféremment utilisé soit pour qualifier une ville sans fonction particulière ou une capitale qu'elle soit régionale ou nationale, soit encore pour présenter une aire urbaine de taille variable, évoluant dans la mouvance d'une ville-centre exerçant des fonctions internationales plus ou moins affirmées. CRDP-thém@docMétropoles européennes, Lille, 2002.
} 
intenses. Sa performance logistique (convergence multimodale) résulte de la conjonction de deux paramètres fondamentaux : une grande accessibilité, elle-même conditionnée par un haut niveau d'équipement en modes de transport rapide parmi lesquels l'autoroute, le TGV ou encore l'aéroport pivot (hub) et une interconnexion complète et réussie entre les différents moyens de communication permettant de gérer efficacement la liaison entre réseaux locaux, régionaux, nationaux et internationaux.

- Des attributs directionnels: la métropole se caractérise par ses activités de commandement. C'est une place financière reconnue qui attire des sièges sociaux d'entreprises nationales et des filiales de compagnies étrangères, un foyer d'innovation, le centre d'un secteur tertiaire industriel périproductif dynamique qui multiplie les services aux entreprises (Joignaux, 1999) et qui se distingue du tertiaire banal présent dans toute ville. L'activité industrielle y a une dimension internationale ou européenne. Pour C. Lacour et S. Puissant (1999), la métropolisation peut être interprétée comme la traduction urbaine de la mondialisation. Comme leur nom l'indique, la présence marquée d'emplois métropolitains supérieurs (EMS) ${ }^{6}$ reflète la métropolisation des espaces. Par ailleurs, grâce au degré de qualification et à la diversité des opportunités d'emploi qu'elle offre, la métropole attire des migrations alternantes d'actifs importantes à partir des espaces qui lui sont contigus et à partir d'espaces connexes, à savoir des territoires plus éloignés auxquels la métropole est bien reliée.

- Des attributs identitaires: la métropole est une ville vitrine qui reflète une image forte d'elle-même au-delà des frontières nationales grâce à une qualité urbanistique reconnue, de solides atouts environnementaux, patrimoniaux et culturels (cadre naturel, vestiges du passé, musées, opéras, festivals), la présence d'universités et d'écoles de renommée mondiale et l'organisation de salons, foires ou congrès à vocation internationale ${ }^{7}$.

- À ces trois premiers attributs ${ }^{8}$, il convient d'en ajouter un quatrième relevant de la coordination des acteurs. Les

\footnotetext{
${ }^{6}$ Selon la définition de l'INSEE, il existe onze fonctions métropolitaines supérieures rassemblant différents types d'emplois dans les services aux entreprises, la recherche, le commerce, le secteur des banques et assurances, l'art, la gestion dans l'industrie, le transport, le commerce dans l'industrie, les télécommunications, l'information et l'informatique dans l'industrie.

7 On pourrait compléter cette approche par celle de C. Gachelin (1992) qui liste les dix commandements d'une métropole réussie et recense les effets de seuil, quantitatifs pour les populations, qualitatifs pour les équipements : le choix de l'investissement haut de gamme, une nouvelle image urbaine, l'accessibilité, la mise en place d'une nouvelle structure spatiale, la capacité à gérer le grand événementiel, l'existence d'un consensus et d'un pouvoir métropolitain, la fonction internationale, les dynamiques induites par les partenariats public-privé et l'implication de l'université dans le développement, la gestion de la métropolisation par une réflexion sur des schémas évolutifs.

8 Développés par CRDP-thém@doc-Métropoles européennes, Lille, 2002.
}

métropoles constituent en effet des points d'articulation et d'interface au sein de différents réseaux. Trois types de réseaux peuvent être distingués : des réseaux d'entreprises, des réseaux de transport, communication, information et innovation, enfin des réseaux de socialisation (Derycke, 1999). Ces derniers revêtent un caractère central en matière de coordination. La métropole est la tête de réseau d'un système urbain régional assez fortement hiérarchisé, comprenant des grandes villes, des villes moyennes et des petites villes en interaction. La mise en réseau, essentielle, implique une recherche de cohésion dans le domaine politique et institutionnel qui nécessite des efforts de coordination, externe et interne. La coordination des acteurs contribue à enclencher une taille critique à même de faire exister un territoire métropolitain. Ainsi, la gouvernance des territoires constitue un enjeu du déploiement des processus métropolitains (voir (Paris, 2004) ou encore (Ginet, 2004) ${ }^{9}$ ). La métropole est ainsi un territoire sur lequel, dépassant les antagonismes locaux, les acteurs partagent une vision commune de leur devenir. Cette dernière constitue la base de leurs anticipations et de leurs actions. Un processus de métropolisation ne peut donc avoir lieu sans un consensus large permettant la coordination d'acteurs et une légitimité de la ville-centre sur un territoire rayonnant au-delà de l'agglomération elle-même.

Ce quatrième attribut alimente les trois premiers, dans la mesure où il influe sur l'identité du territoire. Cette dernière peut aussi renforcer l'attractivité économique du territoire, ainsi qu'être à l'origine d'un renforcement des attributs logistiques par un besoin de gestion de flux croissants.

\subsection{La métropolisation autour de l'agglomération rémoise : un processus émergent}

Au regard des critères évoqués, l'agglomération rémoise ne peut être qualifiée actuellement de métropole même si, à l'instar de Lille-Métropole, elle s'est rebaptisée ReimsMétropole le 3 mai 2005. Tout au plus peut-on évoquer l'émergence d'une métropole polynucléaire (Brillaud, 2004) autour de Reims entourée de sept aires urbaines petites et moyennes ${ }^{10}$.

En matière d'attributs logistiques, l'agglomération rémoise, positionnée au croisement de 1'A4 (Paris-Strasbourg), de l'A26 (Troyes-Calais) et de l'A34 en voie d'achèvement (Reims-Charleville-Belgique), est caractérisée par une bonne accessibilité autoroutière (Fig. 1). Toutefois, elle est restée longtemps à l'écart des principaux

\footnotetext{
9 Analysant les exemples d'Anvers et de Liège, ce dernier auteur associe processus de métropolisation et capacité des acteurs à s'entendre et à trouver des compromis afin de se repositionner après une crise grave.

${ }^{10}$ Il s'agit des villes de Châlons-en-Champagne et Épernay dans la Marne, de villes de l'Aisne comme Château-Thierry, Laon et Soissons, et de Charleville-Mézières et Rethel dans les Ardennes. Ces villes sont donc situées sur un territoire transrégional.
} 


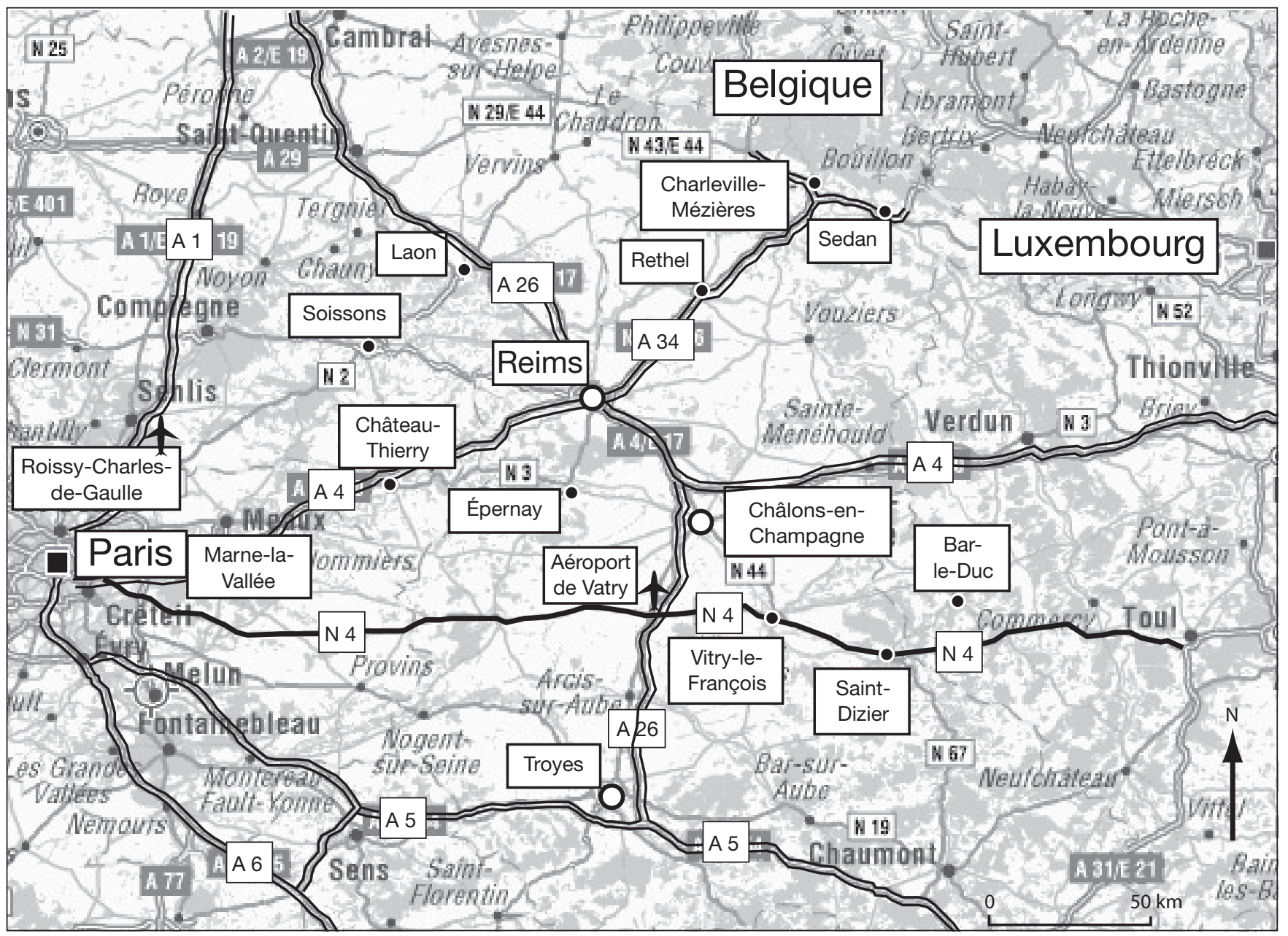

Fig.1 Les principaux axes routiers et autoroutiers autour de l'agglomération rémoise

axes de circulation routière, notamment de la RN4 qui traverse la Champagne-Ardenne en reliant Paris à Nancy et à Strasbourg. Cette situation est également observée en matière de transport ferroviaire, puisque Reims ne se situe pas sur la grande radiale Paris-Strasbourg qui passe par la vallée de la Marne. Cela ne l'empêche toutefois pas de bénéficier actuellement d'une bonne desserte ferroviaire vers Paris, tout comme les villes proches. L'accès aux villes de province est cependant jugé moins satisfaisant en raison d'une rupture de charge à Paris. Enfin, dans le domaine du transport aérien, il manque une véritable plateforme aéroportuaire. Le développement de l'aéroport international de Vatry est encore trop limité, notamment en matière de transport de passagers, pour pouvoir remplir ce rôle ${ }^{11}$.

En ce qui concerne les attributs directionnels et plus particulièrement en matière de sièges sociaux, l'agglomération

\footnotetext{
${ }^{11}$ L'aéroport de Vatry situé au Sud de Châlons-en-Champagne, à 70 km de Reims, est initialement dédié au fret. Quelques vols charters y sont actuellement réalisés. Sa vocation mixte a été renforcée par la construction d'une aérogare passagers.
}

rémoise est caractérisée par un faible taux d'autonomie qui ne dépassait pas $59,5 \%$ en $2000^{12}$.

Par ailleurs, si elle est caractérisée par une relative importance du tertiaire $(78,8 \%$ des emplois contre $77,7 \%$ pour les unités urbaines de 80000 à 150000 emplois) et par leur forte croissance entre les deux recensements $(+16,7 \%$ contre $13,9 \%$ pour la moyenne des mêmes unités urbaines), l'analyse de la structure des activités tertiaires dans une nomenclature plus fine indique que les services aux entreprises y sont légèrement sous-représentés à l'inverse des services financiers ${ }^{13}$.

\footnotetext{
${ }^{12}$ Le taux d'autonomie mesure le rapport entre le nombre d'emplois dans les établissements sièges et le nombre d'emplois dans l'ensemble des établissements de l'intercommunalité. Pour comparaison, les intercommunalités de Lens-Liévin et Hénin-Carvin dans le Nord qui présentent également une structure urbaine très polarisée ont des taux d'autonomie respectifs de $65,6 \%$ et de $64,8 \%$ (INSEE, 2002).

${ }^{13}$ Les services aux entreprises représentent $11,70 \%$ des emplois en 1999 à Reims contre $11,72 \%$ au Mans 12,04 \% à Tours et 16,97\% à Orléans. Les activités financières regroupent $4,21 \%$ des emplois à Reims contre 5,34\% au Mans, 3,39\% à Tours et 3,65\% à Orléans. Cette présence plus importante des activités financières ne confère cependant pas un pouvoir décisionnaire en la matière, dans la mesure où les décisions de financement peuvent être prises à des échelons hiérarchiques supérieurs.
} 
L'analyse des EMS dans l'aire urbaine de Reims fournit également des résultats contrastés. En effet, si ces EMS sont de façon globale relativement bien représentés comparativement à la taille de la ville, notamment dans la fonction recherche ( $22 \%$ des EMS contre 15,5\% pour la France métropolitaine) et s'ils sont également en forte croissance ${ }^{14}$ entre 1990 et 1999, ils sont en revanche sous représentés par rapport à la France métropolitaine dans les services aux entreprises ${ }^{15}$.

L'analyse des migrations alternantes en ChampagneArdenne recensées par l'INSEE indique également un poids important de l'aire urbaine de Reims qui attire des migrants en provenance des aires urbaines champardennaises telles que Châlons-en-Champagne (17,5\% des flux totaux), Épernay $(16 \%)$, Rethel $(12,7 \%)$, ainsi que, hors ChampagneArdenne, en provenance de Paris (13,7\%), Laon $(6,4 \%)$, Soissons $(3,4 \%)$ et Château-Thierry $(2,4 \%)$. Par ailleurs, la part au sein des actifs de ceux qui vont travailler dans l'agglomération rémoise est non négligeable pour certaines aires urbaines comme Rethel (14,98\%), Épernay (5,58\%) et Châlons-en-Champagne (3,14\%) (tableau 1).

L'influence de Reims dépasse donc les seules limites de son aire urbaine, puisque cette dernière attire une partie des actifs des aires urbaines situées à proximité. Reims semble ainsi polariser son espace local et entretenir des rapports de proximité avec les villes de son environnement. À l'échelle des déplacements domicile-travail, on voit donc apparaître des modes d'organisation de type christallerien ${ }^{16}$ autour de

$1422,3 \%$, i.e. un pourcentage plus important que pour la France de province $(17,9 \%)$ ou les grandes villes de France $(18,3 \%)$.

$1523,8 \%$ contre $31,6 \%$ pour la France métropolitaine.

16 Le modèle de Walter Christaller (1933) est un modèle caractérisant l'armature urbaine d'un territoire et dérive de la théorie des lieux centraux qui rend compte de la taille, de l'espacement et du nombre des villes. Le modèle décrit l'organisation hiérarchisée d'un réseau de villes selon le niveau des services qu'elles offrent, et leur disposition spatiale régulière aux sommets de triangles équilatéraux ou au centre d'hexagones.
Reims (Gilli, 2002). À la lecture des relations qu'entretient Reims avec les aires urbaines qui l'entourent, il semblerait que l'éventuel processus de métropolisation doive être envisagé comme celui d'une métropole polynucléaire autour de Reims sur des territoires multipolarisés. Toutefois, l'aire urbaine de Reims est également influencée par la proximité de Paris. Cette influence peut être étudiée à partir de l'analyse des flux domicile-travail partant de l'aire urbaine de Reims à destination des zones d'emploi ${ }^{17}$ de l'Île-de-France (tableau 2), le découpage en zones d'emploi permettant de mieux repérer l'orientation géographique des flux.

Au total, on recense 1584 déplacements domicile-travail partant de l'aire urbaine de Reims vers les zones d'emploi de l'Île-de-France, ce qui représente 14,2 \% du total des déplacements domicile-travail quittant l'aire urbaine de Reims. Ces flux se dirigent majoritairement vers la zone d'emploi de Paris (commune de Paris, 41,3\%), puis vers la zone d'emploi de Nanterre $(12,5 \%)^{18}$, alors que celle-ci se situe géographiquement à l'opposé du Bassin parisien par rapport à Reims. La zone d'emploi de Saint-Denis, située à l'Est de Paris, n'accueille que 7,6 \% des flux de migrants alternants. En outre, les déplacements domicile-travail entre l'aire urbaine de Reims et la ville nouvelle de Marne-la-Vallée sont limités ${ }^{19}$. Enfin, l'aire urbaine de Reims envoie propor-

\footnotetext{
17 Une zone d'emploi est un espace géographique à l'intérieur duquel la plupart des actifs résident et travaillent. Ce découpage est effectué conjointement par l'INSEE et les services statistiques du ministère du Travail.

18 Nanterre accueille une partie du quartier d'affaires de la Défense comme Puteaux et Courbevoie, ce qui explique l'importance des déplacements vers cette commune. Plus de neuf cents sociétés, dont souvent des sièges sociaux, sont installées dans le quartier de la Défense.

${ }^{19}$ En effet, le découpage en zones d'emploi ne permet pas de faire apparaître de façon nette la ville nouvelle de Marne-la-Vallée, qui, nous y reviendrons, sera directement connectée à Bezannes lors de la mise en place de la ligne à grande vitesse.
}

Tableau 1

Répartition des flux de migrations alternantes allant vers l'aire urbaine de Reims en 1999

\begin{tabular}{lrr}
\hline & & Migrations vers l'aire urbaine de Reims \\
\cline { 2 - 3 } Origine : aire urbaine de & Nombre & Répartition \\
\hline Châlons-en-Champagne & 1034 & $17,5 \%$ \\
Épernay & 944 & $16,0 \%$ \\
Paris & 807 & $13,7 \%$ \\
Rethel & 750 & $12,7 \%$ \\
Laon & 378 & $6,4 \%$ \\
Charleville-Mézières & 291 & $4,9 \%$ \\
Soissons & 198 & $3,4 \%$ \\
Château-Thierry & 144 & $2,4 \%$ \\
Vitry-le-François & 125 & $2,1 \%$ \\
Troyes & 98 & $0,73 \%$ \\
Dijon & $63 \%$ & $1,95 \%$ \\
Lille & $63 \%$ & $1,11 \%$ \\
Autres & $0,95 \%$ & $1,1 \%$ \\
Total des migrations alternantes vers l'aire urbaine de Reims & 1014 & $1,1 \%$
\end{tabular}

D'après le Recensement général de la population de 1999 (INSEE, 1999) 
Tableau 2

Flux de migrations alternantes au départ de l'aire urbaine de Reims vers les zones d'emploi de l'Île-de-France en 1999

\begin{tabular}{|c|c|c|c|}
\hline \multirow{2}{*}{ Destination : zone d'emploi de } & \multirow{2}{*}{ Emplois dans la zone } & \multicolumn{2}{|c|}{ Migrations depuis l'aire urbaine de Reims } \\
\hline & & Nombre & Répartition \\
\hline Paris & 1600815 & 654 & $41,3 \%$ \\
\hline Nanterre & 760575 & 198 & $12,5 \%$ \\
\hline Saint-Denis & 389159 & 120 & $7,6 \%$ \\
\hline Montreuil & 311068 & 103 & $6,5 \%$ \\
\hline Créteil & 267088 & 75 & $4,7 \%$ \\
\hline Boulogne-Billancourt & 361117 & 74 & $4,6 \%$ \\
\hline Versailles & 288494 & 52 & $3,3 \%$ \\
\hline Meaux & 44602 & 46 & $2,9 \%$ \\
\hline Orly & 158366 & 39 & $2,5 \%$ \\
\hline Vitry-sur-Seine & 130668 & 38 & $2,4 \%$ \\
\hline Roissy-en-France & 49580 & 37 & $2,3 \%$ \\
\hline Melun & 83620 & 28 & $1,8 \%$ \\
\hline Cergy & 117347 & 27 & $1,7 \%$ \\
\hline Lagny-sur-Marne & 36555 & 22 & $1,4 \%$ \\
\hline Autres & - & 71 & $4,5 \%$ \\
\hline $\begin{array}{l}\text { Total des migrations alternantes vers les zones d'emploi } \\
\text { d'Île-de-France }\end{array}$ & - & 1584 & $100,0 \%$ \\
\hline
\end{tabular}

D’après le Recensement général de la population de 1999 (INSEE, 1999)

tionnellement moins d'actifs (et de moins en moins entre 1990 et 1999) vers les zones d'emplois de l'Île-de-France que d'autres aires à proximité de Paris comme celle de Troyes. Ainsi, l'aire urbaine de Reims semble relativement indépendante de Paris.

En termes d'attributs identitaires, Reims bénéficie d'une réelle notoriété en raison de l'importance de ses attributs touristiques et de la renommée du Champagne. Sa notoriété pourrait toutefois être encore plus forte, compte tenu de la qualité de son patrimoine (Bazin et al., 2004). Reims dispose d'un centre des Congrès dont l'activité se concentre néanmoins essentiellement sur des congrès et conventions d'entreprises, en grande majorité nationaux (Reims champagne congrès expo, 2003).

Un pôle d'enseignement supérieur développé et de qualité fait également partie des attributs identitaires. Dans l'agglomération rémoise, c'est le cas de l'École de Commerce de Reims (Reims Management School) et de l'Université de ReimsChampagne-Ardenne (URCA) avec ses 21424 étudiants, dont $87 \%$ à Reims ${ }^{20}$. Par ailleurs, l'Université et les organismes de recherche nationaux implantés en région participent également au développement de pôles d'excellence. C'est le cas dans le domaine de la valorisation des agro-ressources, puisqu'en coo-

20 Toutefois, les campus rémois de l'Université de Reims-ChampagneArdenne n'accueillent pas l'ensemble des étudiants de la Région. Ainsi les étudiants ardennais vont souvent poursuivre leurs études à Lille, les étudiants hauts-marnais à Nancy ou à Dijon. Les étudiants troyens restent à Troyes le plus souvent ou s'orientent vers la poursuite d'études à Dijon ou à Paris. En outre, la structure déséquilibrée de l'offre de l'URCA (importance du 1er cycle et, inversement, faiblesse du $3^{\mathrm{e}}$ cycle) peut conduire à relativiser sa capacité à produire des emplois hautement qualifiés, symbole d'une métropolisation. pération avec la région picarde, la région ChampagneArdenne vient d'obtenir le label de pôle de compétitivité au niveau mondial. Ce label devrait permettre de soutenir l'activité de recherche, de conforter la notoriété de l'URCA dans ce domaine et de développer également la dimension européenne, voire même internationale qui fait actuellement défaut à l'agglomération rémoise (Rozenblat et Cicille, 2003).

Enfin si l'on analyse le degré de coordination entre acteurs, celui-ci semble actuellement encore faible. Les différentes tentatives pour faire émerger une coopération des agglomérations situées autour de Reims - création dans les années 1960 d'une Zone d'appui nord-champenoise (ZANC) (CESR, 2003), reconnaissance dans les années 1980 de la nécessité d'un fonctionnement en réseau pour tendre vers une métropole polycentrique, loi Pasqua de 1995, ou enfin, au début des années 2000, nouvelle tentative dans le cadre du SRADT ${ }^{21}$ — n'avaient pas abouti au début de l'année 2006. Si Jean-Louis Schneiter, Maire de Reims, déclarait: Châlons-en-Champagne, Charleville-Mézières, Sedan et Château-Thierry pourraient s'associer - en fonction de modalités qui restent à définir - pour participer à la formalisation de pôles urbains éclatés mais solidaires, et constituer une métropole particulière polycentrique, à l'Est $d u$ Bassin parisien (AUDRR, 2004), force est de constater que, jusqu'à présent, cette volonté ne s'était pas traduite

\footnotetext{
${ }^{21}$ Dans le volume II du Schéma régional d'aménagement et de développement du territoire, adopté le 16 octobre 2000, intitulé Construire l'avenir de la Champagne-Ardenne, il est fait référence explicite à la métropole dans l'axe III : le rayonnement de la Région passe également par l'identification d'une grande métropole. Reims, ville historique et de renom international, n'est pas au rang des grandes agglomérations européennes.
} 
dans les faits. Ainsi dans l'appel à coopération de la DATAR dans le cadre de la métropolisation, Reims n'a pas fait acte de candidature en association avec les villes de la Champagne-Ardenne, mais en coopération avec l'ensemble des villes du Bassin parisien ${ }^{22}$. Toutefois, au début de 2005 a été initié un projet de réseau de villes, lequel comprend actuellement dix villes ${ }^{23}$ situées en région et hors région (Picardie), pour en renforcer les complémentarités.

Reims et Épernay se revendiquent ainsi toutes deux capitale du Champagne. Capitale administrative, Châlons-enChampagne souhaiterait une décentralisation plus forte de l'université et se tourne vers Nancy et Paris. Capitale économique, Reims seule n'a pas le poids nécessaire pour être une métropole. De plus, les autres villes ne lui confèrent pas l'entière légitimité qui lui permettrait de coordonner des projets métropolitains. De la même façon, la Chambre de commerce et d'industrie de Reims-Épernay et celle de Châlons-en-Champagne n'ont pas donné suite à l'idée de fusion qui leur aurait permis de définir une orientation commune en matière économique. Reims et Châlons-en-Champagne ont également chacune leur propre agence de développement économique. Le poids démographique aujourd'hui encore insuffisant de Reims explique en partie la faible reconnaissance de sa légitimité à jouer le rôle de métropole.

La fragmentation des politiques et des objectifs limite ainsi l'influence de Reims, qui n'arrive qu'au vingt-neuvième rang des agglomérations du fait de sa compacité, même si elle est la douzième ville de France ${ }^{24}$. À cette taille critique insuffisante il faut ajouter la difficulté persistante de l'agglomération à s'affirmer comme leader de l'intercommunalité à l'échelle du Pays en raison de conflits dans des domaines sensibles tels que le logement social, le développement des commerces ou encore l'organisation intercommunale.

Ainsi, le processus de métropolisation est encore embryonnaire autour de Reims, ville qui souffre d'une taille moyenne, qui est privée du statut de capitale régionale et qui est caractérisée par un relatif conservatisme en raison de la rente que lui confère le champagne. Cependant, l'arrivée de la ligne à grande vitesse nous semble pouvoir, sous certaines conditions, favoriser son développement. Pour ce faire, Reims devra composer avec ses handicaps et alimenter la dynamique des espaces contigus, si elle veut en bénéficier. Ce positionnement doit se réaliser concrètement par des projets coordonnés autour des infrastructures aéroportuaires et

\footnotetext{
${ }^{22}$ Le projet n'a d'ailleurs pas été retenu par la DATAR.

${ }^{23}$ Reims, Châlons-en-Champagne, Épernay et Vitry-le-François dans la Marne, Rethel, Charleville-Mézières et Sedan dans les Ardennes, ChâteauThierry, Laon et Soissons dans l'Aisne.

${ }^{24}$ La ville-centre regroupe à elle seule $87 \%$ de la population totale, soit plus de 187000 sur 215581 habitants, contre $75 \%$ au Mans, $51 \%$ à Toulouse, $42 \%$ à Orléans, $22 \%$ à Paris et $12 \%$ à Valenciennes. Le pays rémois composé de 137 communes regroupe seulement 252000 habitants.
}

routières, des dessertes TER, de l'université, du pôle de compétitivité, ou encore du rayonnement culturel ${ }^{25}$.

\section{L'arrivée de la ligne à grande vitesse dans l'agglomération rémoise : vers un processus de métropolisation?}

Si l'arrivée d'une ligne à grande vitesse peut contribuer à accélérer le pouvoir d'attraction des espaces desservis, elle peut également être à l'origine d'une détérioration de cette attractivité en accroissant certaines formes de dépendance. Toutefois, autour de l'agglomération rémoise et pour peu que les acteurs publics s'en emparent, elle peut être un outil au service du processus de métropolisation.

\subsection{L'arrivée de la grande vitesse: métropolisation versus satellisation?}

Les attributs métropolitains listés précédemment sont plus ou moins susceptibles d'être affectés par l'arrivée de la grande vitesse sur un territoire.

Pour ce qui est des attributs logistiques, la grande vitesse permet incontestablement de renforcer la position de concentration et de redistribution des flux en favorisant l'accessibilité. Elle favorise ainsi le mouvement de polarisation des activités sur les territoires, lequel peut contribuer à un processus de métropolisation si elle est complétée par une bonne accessibilité routière, autoroutière et aérienne. Dans ce domaine, les effets de la grande vitesse dépendent de la qualité de l'irrigation par la desserte ferroviaire et routière du territoire environnant la ville desservie.

En ce qui concerne les attributs directionnels, la grande vitesse permet de favoriser l'accessibilité du territoire desservi aux centres de décisions extérieurs. Lorsque ce territoire est doté d'un pouvoir important d'attractivité et concentre déjà une activité directionnelle, la grande vitesse peut y favoriser également une délocalisation de sièges ${ }^{26}$. Par ailleurs, la grande vitesse permet aux entreprises de rencontrer plus facilement leurs clients et inversement, et ouvre la concurrence des entreprises locales aux entreprises extérieures. Si cette concurrence peut constituer un danger, elle peut également être facteur de dynamisme et d'innovation sur le territoire desservi. La grande vitesse peut faciliter les connexions existant avec d' autres centres (nationaux et internationaux) spécialisés dans le développement de nouvelles technologies et favoriser ainsi le développement des pôles de

\footnotetext{
25 Les maires de Reims, Châlons-en-Champagne, Épernay et Soissons, réunis en table ronde à Reims le 23 juin 2006 dans le cadre du colloque $L a$ Champagne-Ardenne et la Picardie face aux défis de l'attractivité et de la compétitivité des territoires, se sont prononcés pour ces priorités.

26 À elle seule, l'arrivée du TGV n'est évidemment pas suffisante pour entraîner une telle délocalisation.
} 
compétences reconnus. De même, si un tissu industriel dynamique permettant d'alimenter le secteur des services aux entreprises existe, la grande vitesse peut favoriser son développement notamment en facilitant le recrutement de cadres et de chercheurs.

Si l'on observe les attributs identitaires, la présence d'une ligne à grande vitesse véhicule une image de modernité. Même si cet effet s'est atténué depuis les premières mises en service, elle reste un atout dans la compétition entre les villes. Le TGV confère une image de progrès aux yeux des acteurs extérieurs. Il améliore également la représentation que les acteurs locaux et la population ont de leur territoire. Cela étant, les effets du TGV pour les entreprises localisées se limitent parfois à cet effet d'image. Ainsi, si certaines entreprises de services considèrent le TGV-Nord comme un formidable facteur de développement pour la région, elles ne l'utilisent pas dans leurs pratiques de déplacement (Colletis-Wahl et Burmeister, 1996). Il existe souvent un écart entre l'image véhiculée par le TGV et son usage réel, ainsi que le confirment les entretiens que nous avons réalisés auprès des grandes entreprises de Champagne-Ardenne (Bazin et al., 2006). Enfin, si les infrastructures hôtelières et le patrimoine l'autorisent, la grande vitesse peut également permettre d'alimenter l'activité des centres des Congrès et d'élargir leur notoriété nationale, voire internationale, comme le montrent les expériences mancelle et tourangelle 27 .

Par ailleurs, le rapprochement, en termes de distancetemps, permis par le TGV vers les zones d'emploi d'Île-deFrance ne se traduit systématiquement ni par un attrait de nouvelles populations (Bazin et al., 2006), ni par un développement des migrations alternantes lointaines: Ces déplacements restent marginaux et leur nombre n'évolue guère (INSEE-IAURIF, 2003 ; 96). Seules certaines agglomérations desservies par le TGV ont connu une croissance de leurs actifs travaillant à Paris. C'est le cas de Tours, Nantes, Vendôme. Le nombre de migrants alternants en direction de l'Île-de-France à Saint-Pierre-des-Corps, Poitiers, Angoulême et Bordeaux a diminué ${ }^{28}$. Ainsi, l'analyse des expériences passées montre que, si l'influence sur les attributs ne peut être contestée, la réalité des effets est loin d'être systématique.

\footnotetext{
27 Au Mans, avant 1989, année précédant l'arrivée du TGV, 15 manifestations de congrès avaient été organisées (Bellanger, 1991). En 1993, on en dénombrait 30, puis 500 en 1995 pour 310 jours d'activité (Amiard, 1997 ; 77), soit un taux d'occupation de l'ordre de $85 \%$. Cette évolution témoigne d'une attractivité nouvelle pour Le Mans et de la reconnaissance par les décideurs de réels atouts. En 1993, soit trois ans après l'arrivée du TGV, $70 \%$ des touristes d'affaires du Mans venaient en TGV. Depuis son inauguration en septembre 1993, le palais des congrès Le Vinci de Tours a accueilli près de 1300 manifestations et plus de 1250000 congressistes et spectateurs, dans le cadre d'une activité liée pour $90 \%$ au tourisme d'affaires (www.francecongres.org).

28 Plus généralement, les résultats du recensement témoignent d'une baisse du nombre d'actifs de province travaillant en Île-de-France et résidant loin, c'est-à-dire au-delà des huit départements limitrophes de cette région (124000 en 1990, 102000 en 1999) (INSEE-IAURIF, 2003 ; 96).
}

Les observations n'indiquent pas non plus une progression des déplacements professionnels liés à la mise en place du TGV-Atlantique (Klein et Claisse, 1997). La conjoncture dégradée du début des années 1990, l'augmentation tarifaire et les problèmes liés au système de réservation ${ }^{29}$ ont eu un effet restrictif sur le trafic ferroviaire, notamment professionnel. Toutefois, l'analyse met en évidence que pour des zones de relative proximité à Paris et pour lesquelles les temps généralisés de déplacements par route et par TGV sont relativement proches, la sensibilité au prix et à la qualité de service devient essentielle (Klein et Claisse, 1997 ; 55). Il est vrai que les voyages pour des durées de séjour de l'ordre de la demi-journée ont connu une évolution différente puisqu'ils croissent de façon extrêmement importante $(+260 \%)$ et que certains secteurs comme le secteur étudeconseil-assistance ou celui des services non marchands sont caractérisés par des évolutions moins défavorables (stabilité pour le premier et croissance importante pour le second). Mais globalement, en termes de mobilité professionnelle, l'offre TGV n'a pas suscité l'apparition ou le développement de nouvelles pratiques de déplacement (Klein et Claisse, $1997 ; 72)$.

Enfin, en termes de coordination des acteurs, un processus de métropolisation ne peut se produire sans un consensus large autour de la ville-centre. En accélérant la prise de conscience de cette nécessité, l'arrivée de la ligne à grande vitesse peut alors être perçue comme un outil de coordination. Cela a été le cas à Lille : Les enjeux du développement métropolitain à partir du milieu des années 1980 ont amené différents mondes à se côtoyer, par exemple au sein du club informel animé par Jean-Paul Bä̈etto, le responsable d'Euralille. La cristallisation des réseaux d'individus autour de projets (bataille du TGV à Lille, Jeux olympiques...) et dans des groupes (Comité Grand Lille) a permis la solidification du consensus métropolitain sur lequel les politiques ont pu ensuite s'appuyer (Paris, $2004 ; 35)$.

À une échelle territoriale plus large, des travaux montrent également que le type d'appropriation régionale de la grande vitesse peut conditionner sa valorisation au travers d'une adhésion (ou non) collective au projet. Ainsi, dans le Nord Pas-de-Calais, P. Menerault (1996 et 1997) a montré comment un grand projet ferroviaire, largement marqué par son empreinte nationale, a pu faire l'objet d'un fort investissement régional, d'une territorialisation de l'infrastructure. De même, l'analyse des cas de la Wallonie et de la Bretagne montre que les choix des acteurs par rapport aux schémas envisagés ont été essentiels pour la mobilisation de ceux-ci (Menerault, 2000).

Les effets de la grande vitesse ne sont d'ailleurs pas liés à la seule présence du TGV, mais aussi à la conjoncture économique de la ville desservie, à sa distance à Paris, à la

\footnotetext{
${ }^{29}$ Socrate, nouveau système de réservation de la SNCF, a conduit à d'importants dysfonctionnements.
} 
tarification, aux fréquences et aux horaires, aux potentialités économiques et sociales locales, ainsi qu'à l'articulation entre le TGV et le réseau de desserte locale. La grande vitesse est un révélateur des forces et faiblesses du territoire. Elle accentue l'attractivité des territoires déjà dotés d'un pouvoir d'attractivité élevé, mais peut au contraire renforcer le déclin des territoires peu attractifs, fragmentés, émiettés. La grande vitesse, en effet, facilite les liens avec les territoires éloignés, lesquels peuvent alimenter les territoires desservis ou les vider. Cette menace d'absorption peut conduire à un appauvrissement des relations internes au territoire, surtout si la grande vitesse les rapproche de façon importante de territoires dotés d'une hyperattractivité comme Paris. Dans ce cas, elle peut déboucher sur une satellisation des territoires desservis par d'autres métropoles ou par une mégapole, satellisation qui peut renforcer la déstructuration et l'éclatement de relations interrégionales déjà fragiles. Mais une telle infrastructure peut aussi déclencher une prise de conscience de ces risques, susciter des dynamiques nouvelles et être ainsi à l'origine d'une réorientation des stratégies menées.

\subsection{L'arrivée de la ligne à grande vitesse \\ dans l'agglomération rémoise : un outil au service du processus de métropolisation}

En 2007, la ligne à grande vitesse est-européenne desservira plusieurs villes de la région Champagne-Ardenne et notamment l'agglomération rémoise qui, par l'intermédiaire de deux gares, bénéficiera de la desserte la plus importante en termes de fréquence.

La gare Champagne-Ardenne, gare nouvelle située à Bezannes à $5 \mathrm{~km}$ au Sud de Reims, a pour vocation de relier la région aux gares TGV d'île-de-France (Massy, Marne-laVallée et aéroport Roissy-Charles-de-Gaulle), lui assurant ainsi une connexion directe aux réseaux à grande vitesse Nord, Ouest et Sud-Ouest sans transiter par Paris. Six allers et retours y sont prévus à destination des gares de Marne-laVallée (en $30 \mathrm{~min})^{30}$ et de Massy (en une heure) et, plus loin, vers l'Ouest et le Sud-Ouest. Trois allers-retours sont prévus à destination de l'aéroport Roissy-Charles-de-Gaulle (en 30 min) et, au-delà, vers le Nord. En outre, deux allersretours à destination de la gare de l'Est parisienne seront également offerts à partir de cette gare.

La gare centrale de Reims, dite gare Clairmarais, accueillera huit allers et retours de rames TGV qui desserviront Paris par la gare de l'Est en 45 min (contre actuellement douze allers-retours en $1 \mathrm{~h} 35$ ). La ligne à grande vitesse diminuera ainsi de façon importante la durée du trajet entre Reims et Paris $(-53 \%)$, plaçant Reims à une distance de

\footnotetext{
${ }^{30}$ Cette liaison ferroviaire, inexistante actuellement, mettra le train en position compétitive par rapport à la voiture puisqu'elle permettra une réduction de moitié des temps de parcours actuels.
}

Paris équivalente à celle qui caractérise les villes de la quatrième zone RATP.

Si la capacité de l'agglomération rémoise à devenir la métropole régionale réside dans sa capacité à se doter et/ou à développer les attributs métropolitains évoqués précédemment, l'arrivée de la ligne à grande vitesse peut y participer à plusieurs égards.

D'un point de vue logistique, la ligne à grande vitesse renforce l'accessibilité de Reims à Paris-Est, à l'Est de l'Île-deFrance et plus généralement aux villes de province connectées au réseau à grande vitesse. En renforçant l'accessibilité à l'aéroport Roissy-Charles-de-Gaulle qui ne sera plus qu'à 30 min de la gare Champagne-Ardenne de Bezannes ${ }^{31}$, elle permettra à l'agglomération rémoise d'être dotée d'un nouvel attribut métropolitain, un aéroport international, attribut qui sera conditionné toutefois par la qualité de la desserte mise en place ${ }^{32}$. Comme le montre l'analyse du cas lillois, La transformation des relations espace-temps entre Lille et Roissy due à l'évolution du système TGV (liaison, temps, fréquences) amène à renouveler la question de l'accessibilité en faisant de Roissy, pour des destinations nationales, un second aéroport lillois (Menerault et Stransky, 1999). L'arrivée de la ligne à grande vitesse est-européenne ouvre également l'ensemble des zones d'emplois de Marne-la-Vallée et Roissy aux entreprises implantées à Reims ${ }^{33}$.

La réorganisation des dessertes TER qui accompagnera cette arrivée a été bien accueillie par l'ensemble des acteurs, car elle permettra à une partie du territoire régional (le centre et le Nord) de bénéficier des dessertes TGV à Reims tout en améliorant la qualité de la desserte locale. En outre, des TER en provenance des Ardennes rejoindront la gare Champagne-Ardenne de Bezannes, permettant une interconnexion entre les TER et la ligne à grande vitesse qui favorisera l'usage de la grande vitesse par une part plus importante des champardennais.

En termes d'attributs directionnels, l'arrivée de la ligne à grande vitesse est-européenne pourrait permettre à l'agglomération rémoise d'attirer de nouvelles entreprises et/ou de développer différentes activités. Elle pourrait conduire en effet l'agglomération rémoise, qui se trouve en marge de l'Île-de-France ${ }^{34}$, à se présenter comme une alternative à

\footnotetext{
${ }^{31}$ En termes de temps de parcours, l'agglomération rémoise sera plus proche de l'aéroport Roissy-Charles-de-Gaulle que les arrondissements parisiens.

${ }^{32} \mathrm{Ce}$ faisant, elle risque d'empêcher le développement d'un véritable aéroport régional.

33 Même si l'accessibilité des emplois de la zone Marne-la-Vallée à partir de la gare TGV n'est pas aisée.

${ }^{34}$ L'Île-de-France est en effet caractérisée par une saturation de l'offre immobilière d'entreprises. En 2002, elle proposait près de mille zones d'activités économiques (ZAE) installées à proximité des zones économiques existantes et des infrastructures de transport, soit 18000 ha accueillant ou susceptibles d'accueillir des activités (IAURIF, 2002). Toutefois, malgré une importance et une variété certaines, l'offre souffre de handicaps. Le segment du haut de gamme connaît une pénurie qui contribue à limiter l'attractivité de l'Île-de-France, notamment en direction des investisseurs nationaux et internationaux.
} 
une localisation dans l'Est de cette région. Cela présuppose que le territoire soit en mesure de proposer des locaux pour ces activités à des niveaux de prix (ou de loyers) attractifs. En matière de bureaux, le quartier d'affaires dit Clairmarais, en cours de réalisation derrière la gare de Reims, pourrait permettre de développer les services aux entreprises ${ }^{35}$. La ligne à grande vitesse est également susceptible d'aider à développer la dimension internationale ou européenne de l'activité industrielle grâce à la proximité renforcée de l'aéroport de Roissy.

Par la création de Reims Champagne Développement (RCD) il y a trois ans, l'agglomération rémoise communique autour des avantages concurrentiels d'une localisation rémoise et de l'arrivée de la ligne à grande vitesse Est. L'agglomération rémoise se situe dans une position très favorable en matière d'avantages comparatifs, tant quantitatifs (coûts des ressources humaines, fiscalité, immobilier ${ }^{36}$ ) que qualitatifs (accessibilité, qualité de vie, disponibilité et qualification de la main-d'œuvre) et ce, quelle que soit l'activité envisagée. Le coût d'une implantation à Reims serait inférieur de plus de $20 \%$ à celui d'une implantation parisienne ${ }^{37}$. Cependant, à court terme, les disponibilités foncières sont limitées pour les locaux d'entreprises. S'il existe de nombreuses ZAE dans l'agglomération rémoise, elles sont presque totalement commercialisées ou ne permettent pas de répondre aux demandes de surfaces importantes. Quelques grandes surfaces ont ainsi été recherchées sans succès par des entreprises voulant s'y installer. Dans la perspective notamment de la mise en service de la ligne à grande vitesse en 2007, les collectivités locales et les acteurs économiques ont pris conscience de la nécessité de prévoir des solutions pour continuer d'accueillir des entreprises. Ainsi en matière de ZAE, plusieurs projets permettent un développement de l'offre à moyen terme ${ }^{38}$.

\footnotetext{
$35 \mathrm{Il}$ devrait accueillir près de $70000 \mathrm{~m}^{2}$ de bureaux, dont près de $25000 \mathrm{~m}^{2}$ sont déjà occupés par des entreprises locales et des administrations.

36 Ainsi les loyers annuels par $\mathrm{m}^{2}$ demandés pour des locaux d'activités à Reims se situent à moins de $40 €$, alors que les loyers fluctuent entre 60 et $100 €$ dans la ville nouvelle de Marne-la-Vallée. En ce qui concerne l'immobilier de bureaux, les loyers annuels par $\mathrm{m}^{2}$ pour des locaux de seconde main s'établissent entre $100 €$ et $130 €$ (pour les constructions les plus récentes autour de la gare centrale de Reims) contre des loyers de $248 €$ dans les arrondissements du Nord-Est de Paris, $170 €$ dans la première couronne et $140 €$ à Marne-la-Vallée. Ces chiffres montrent l'intérêt d'une installation dans l'agglomération par rapport à une localisation francilienne, en particulier pour des activités nécessitant une grande surface de locaux. Par ailleurs, les coûts liés aux locaux ne représentent que $5 \%$ à $15 \%$ du total des coûts (ressources humaines, fiscalité, immobilier) d'une entreprise, les salaires représentant 80 à $90 \%$ de ces coûts. Or, les salariés dans l'agglomération rémoise perçoivent des salaires inférieurs en moyenne d'environ $20 \%$ à ceux pratiqués en Île-de-France.

37 Étude confidentielle commandée par Reims Champagne Développement.

${ }^{38}$ Extension du parc de référence Henri Farman de 15 ha, création de la ZAC Croix-Blandin de 60 ha extensibles à plus long terme par 272 ha. Un parc tertiaire de 90 ha est également en cours d'aménagement à côté de la gare Champagne-Ardenne de Bezannes
}

Si les acteurs se mobilisent pour offrir du foncier et favoriser l'offre immobilière d'entreprise, l'analyse des expériences passées montre que l'implantation de nouveaux établissements à la suite de la mise en service d'une ligne à grande vitesse est souvent limitée. Ainsi la demande émane souvent d'entreprises locales qui profitent d'une nouvelle offre pour agrandir leurs surfaces ou bénéficier de locaux plus adaptés aux standards contemporains (Bazin et al., 2006). Par ailleurs, la création de zones d'activités à proximité des nouvelles gares TGV a rarement débouché sur des succès (Bazin et al., 2006). Dans le cas de la gare Champagne-Ardenne de Bezannes et de sa zone d'activités, la proximité de Reims et le contournement autoroutier Sud de Reims en 2011 apparaissent comme des atouts supplémentaires. Toutefois, la gare reste avant tout un lieu de transit, et l'amélioration de l'accessibilité locale et régionale de ces nouveaux quartiers de gare, nécessaire à leur intégration territoriale, renforce cette fonction de transit et risque de favoriser une dilution des activités dans l'ensemble des pôles des agglomérations, au détriment de leur fonction polarisatrice (Facchinetti-Manonne, 2004 ; 84).

Malgré ces incertitudes, ces projets montrent la mobilisation des acteurs pour lever la contrainte de la relative rareté foncière dans l'agglomération. De ce point de vue, les effets de la ligne à grande vitesse sont donc moins directs et sont conditionnés en partie par les politiques d'accompagnement.

En ce qui concerne les attributs identitaires, l'arrivée de la ligne à grande vitesse permet à Reims d'entrer dans le club des villes TGV. À ce titre, elle adhère à l'association des villes européennes de la grande vitesse. Cette arrivée peut contribuer à développer la dimension européenne et internationale de Reims, notamment en accroissant le caractère international de l'activité de son centre des Congrès en matière de tourisme d'affaires (Scherr, 2004). L'agglomération cherche également à se doter d'infrastructures de haut niveau. C'est le cas du tramway, dont le projet a été voté en février 2005 et dont les travaux devraient débuter en 2008, ainsi que du stade, qui est en cours de reconstruction. De plus, des actions de valorisation du patrimoine culturel et touristique sont en cours (nouvelle signalétique ou encore mise en lumière de la cathédrale et du théâtre). Si ces actions ne sont pas toutes directement liées à la ligne à grande vitesse, celle-ci a favorisé la prise de conscience de la nécessité de renouveler l'image de la ville.

Enfin, en termes de coordination, un consensus s'est dégagé entre les acteurs sur le fait que l'arrivée de la ligne à grande vitesse doit être utilisée comme un outil au service du territoire et de son développement, aussi bien au sein de l'agglomération qu'entre l'agglomération et son environnement. Ce consensus ne débouche cependant pas encore aujourd'hui sur une véritable coordination qui permettrait de reconnaître le rôle de Reims comme moteur du processus de métropolisation. Cette coordination, au service d'une dynamique territoriale doit permettre de conforter chaque pôle 
dans son identité et sa complémentarité avec Reims : Épernay comme capitale du Champagne, Châlons-enChampagne comme capitale administrative, Reims comme capitale économique. Les villes de l'Aisne (ChâteauThierry, Soissons, Laon) et Rethel permettent l'accueil de populations dans un cadre urbain et périurbain de qualité, avec possibilité d'accéder aux services de bon niveau présents dans l'agglomération rémoise (université, hôpitaux, infrastructures culturelles et événementielles).

À ce jour, l'arrivée de la ligne à grande vitesse suscite des coopérations à quatre échelles territoriales différentes :

- une première, située autour de l'agglomération rémoise avec les villes d'Épernay et de Châlons-en-Champagne, avec des projets qui ne sont cependant pas aujourd'hui concrétisés ;

- une deuxième, plus large, constituée d'un réseau de villes réactivé récemment, sur une échelle territoriale qualifiée de marnardaisne qui cherche à fédérer les territoires de la Marne, des Ardennes et de l'Aisne ;

- une troisième, à l'échelle départementale, avec des groupes de réflexion à l'initiative du Conseil général de la Marne constitués de différents acteurs publics et privés travaillant sur des thèmes tels que le tourisme, l'attraction de population et d'entreprises, ou encore l'enseignement supérieur ; - une dernière, à l'échelle régionale, avec une concertation, jugée fructueuse par l'ensemble des acteurs concernés, visant à réorganiser la future desserte TER en articulation avec le TGV; de même, en organisant la restitution des travaux relatifs à l'analyse prospective de l'arrivée du TGV en Champagne-Ardenne (qu'il a financés) devant de nombreux acteurs publics locaux et ce, dans les quatre départements de la Région, le Conseil régional a participé indirectement à la prise de conscience de la nécessaire coordination et stimulé la volonté d'utiliser la grande vitesse au service d'une identité nouvelle pour la région.
Une certaine dynamique de réflexion s'est donc enclenchée autour de l'arrivée du TGV. Celle-ci doit se renforcer, s'institutionnaliser et se concrétiser par des projets. C'est le prix à payer pour faire de la grande vitesse un véritable outil de coordination au service d'un processus de métropolisation autour de l'agglomération rémoise.

\section{Conclusion}

Avant l'arrivée de la ligne à grande vitesse, Reims possède un potentiel d'attractivité limité, voire très limité au niveau international, et ne peut être considérée comme une véritable métropole. L'arrivée de la ligne en 2007 conférera une meilleure accessibilité aux territoires desservis. Elle pourrait également permettre de conforter certains attributs métropolitains en favorisant les connexions avec d'autres métropoles. Elle peut également faciliter le recrutement de cadres et de chercheurs dans le secteur tertiaire. Enfin, elle véhicule une image de modernité. Cependant, Reims ne peut prétendre seule à la course à la métropolisation et doit s'appuyer sur les aires urbaines environnantes pour constituer une métropole polynucléaire. Elle doit lutter contre la fragmentation des politiques et des objectifs, qui limite son influence et rend stériles, car non coordonnés, les efforts de territoires interdépendants, commandés par une communauté de destin. De ce point de vue la grande vitesse suscite des dynamiques nouvelles. Vécue initialement comme un choc exogène sur lequel le territoire n'a qu'une emprise limitée, elle est en effet à l'origine et/ou contribue à une évolution des stratégies territoriales. En accélérant la prise de conscience de la nécessité d'une coordination entre les acteurs locaux, l'arrivée de la ligne à grande vitesse peut donc constituer un outil de concertation, permettant une coordination, au service du processus de métropolisation. 


\section{Abridged version}

\section{Introduction}

By reducing the journey times between certain urban zones in the Champagne-Ardenne region and Paris, the EastEuropean high-speed line will bring Rheims, which is the main conurbation it serves in Champagne-Ardenne, closer to Paris and its suburbs as well as areas in the East (Strasbourg, Germany, Switzerland). By interconnections it will also bring some provincial towns which are connected to the high-speed rail network closer, in particular Lille, Nantes, Rennes and Bordeaux. The aim of this paper is to assess to what extent the high-speed line might encourage a metropolization process by improving the accessibility of the conurbation of Rheims. The political and institutional players in Rheims are counting on this better accessibility to increase the attractiveness of the conurbation and reinforce the metropolization process around it. After demonstrating that this process is still at an embryonic stage, the paper will show that the arrival of high-speed rail may, under certain conditions, influence some criteria of metropolization, in particular coordination between local actors.

\section{The process of metropolization around the Rheims conurbation}

There is a scientific consensus which considers metropolization to be a set of dynamic processes which transform a city into a metropolis (Derycke, 1999; 2), but a precise definition is still lacking. Many attempts at clarification have been made (for example (Corade, 1994)) which have produced a certain amount of consensus about the attributes of a metropolis. This paper will adopt the definition proposed by G. Wackermann (2000; 65): The true metropolis is a major urban centre with all or almost all of the urban functions at the highest level of quality [...]. It is a centre for leadership activities in extremely varied spheres, plays a role in the organization of global space and exerts its influence over a territory which is comparatively large and continuous. The attributes of a metropolis are thus as follows.

- Logistical attributes: a metropolis is a major nodal point. It generates flows, including immaterial flows, but is also a centre which concentrates and redistributes large-scale flows of various types (human, goods, data and capital).

- Leadership attributes: a metropolis is characterized by leadership activities. It is a recognized financial centre which attracts the head offices of national companies and the subsidiaries of foreign companies. It is a centre for innovation and has a dynamic periproductive industrial tertiary sector which differs from the ordinary of tertiary sector present in an ordinary city. The large-scale presence of senior executive jobs reflects the existence of these attributes.

- Identity attributes: a metropolis is a showcase city which sends out a strong image of itself beyond national boundaries thanks to a recognized high quality urban environment, an important heritage, cultural assets, the presence of internationally renowned universities and schools and the organization of international shows, fairs or congresses.

- There is a fourth attribute in addition to these three, which involves the coordination of actors. Metropolises are nodes where different networks, in particular socialization networks, link up and interface. The creation of networks is essential and involves an attempt to achieve political and institutional cohesion which requires external and internal coordination efforts. A metropolization process cannot therefore take place without a broad consensus which allows the coordination of actors and gives legitimacy to the central city within a zone whose influence extends beyond the conurbation itself.

If we assume that the metropolization process requires these attributes, in what follows it will be seen that the Rheims conurbation cannot be described as a metropolis, even if, on 3 May 2005, the Rheims conurbation changed its name to Rheims-Métropole in imitation of Lille-Métropole. At most, one can describe the emergence of a polynuclear metropolis (Brillaud, 2004), as the result of the presence around Rheims of seven small and medium-sized urban areas.

\subsection{Logistical attributes}

Although the city has good accessibility by motorway, for a long time the main roads, in particular the RN4 which passes through Champagne-Ardenne and connects Paris to Nancy and Strasbourg (Fig. 1), passed it by. A similar situation applies for rail transport, as Rheims is not on the main radial line between Paris and Strasbourg which passes through the Marne Valley. This does not prevent it from having a good service to Paris now, like all the nearby cities. Access to other provincial cities is, however, less satisfactory because of the need to change trains in Paris. Moreover, as regards air transport, it lacks a major airport. The international airport at Vatry has exhibited a notable increase in freight traffic since its creation in 2000. But, it is still too small, particularly as regards passenger transport, for it to fulfil this role.

\subsection{Leadership attributes}

The ratio between the number of persons employed in head offices and the total workforce in Rheims is low (less than $59.5 \%$ in 2000). This contrasts with two similarly highly centralized intercommunal groupings in the Nord Département, Lens-Liévin and Hénin-Carvin which have 
respective rates of $65.6 \%$ and $64.8 \%$. In addition, although Rheims has a relatively large tertiary sector in terms of the number of jobs which grew considerably between the last two censuses, business services are relatively underrepresented compared with financial services. Likewise, although senior executive jobs are generally speaking fairly well represented in relation to the size of the city, in particular in the research sector, and also increased considerably in number between 1990 and 1999, they are under-represented in business services compared with the rest of metropolitan France.

The importance of the Rheims urban area is also apparent for commuting, as it draws commuters in from the other urban areas in Champagne-Ardenne, such as Châlons-enChampagne, Épernay, Rethel, and, outside ChampagneArdenne, as well as from Paris, Laon, Soissons and ChâteauThierry (Table 1). Rheims appears to hold a central place for the area around it and maintains close ties with its neighbouring cities. Commuting trips reveal a situation around Rheims that complies with Christaller's central place theory (Gilli, 2002). But the urban area of Rheims is also influenced by the closeness of Paris. Commuting trips from the Rheims urban area into the employment zones of the Paris region account for $14.2 \%$ of all commuting trips to outside the Rheims urban area (Table 2). However, it is fairly independent from Paris as proportionately fewer of its workers (and less in 1999 than in 1990) commute into the Paris Region than is the case for other areas that are close to Paris, such as Troyes for example.

\subsection{Identity attributes}

Rheims has considerable fame due to its tourist attractions and the reputation of Champagne. It has a congress centre which mainly organizes national events. Its identity attributes also include a large high quality further education zone. The University and the region's national research institutes also help to develop centres of excellence. This applies in particular to the agro-industries sector, as, in cooperation with the region of Picardy, ChampagneArdenne obtained the label of global competitiveness centre in this area in 2005. However, the European, or even the international dimension, are currently lacking in the Rheims conurbation (Rozenblat and Cicille, 2003).

\subsection{The degree of coordination between actors}

The degree of coordination between players still seems low at present. In the past, various attempts have been made to generate cooperation between the urban areas around Rheims, but unsuccessfully. Thus, in a recent call for cooperation issued by the DATAR with reference to metropolization, Rheims was not a candidate in association with the other cities in Champagne-Ardenne, but in cooperation with all the cities in the Paris Basin. However, in early 2005, a project to produce a network of cities with the aim of reinforcing complementarities was launched. This currently includes ten cities located either within the region or outside it (Picardy). The fragmentation of policies and objectives has limited the influence of Rheims. Furthermore, despite the fact that it is the 12th largest city in France, the conurbation of Rheims is only the 29th French conurbation in terms of size. In addition to this undistinguished position, the conurbation has persistant difficulties in asserting itself as the leader of the communes in the region.

To conclude, the process of metropolization is still at an embryonic stage around Rheims: the city suffers from its moderate size, lacks the status of regional capital and is marked by a degree of conservatism due to its guaranteed income from Champagne.

\section{Will the arrival of the high-speed rail line encourage a process of metropolization?}

In 2007, the East European high-speed rail line will serve several cities in the Champagne-Ardenne region, in particular the conurbation of Rheims whose two stations together will give it the highest service frequencies. The new Champagne-Ardenne station, located $5 \mathrm{~km}$ to the South of Rheims in Bezannes, will have nine return services per day and connect the region to the TGV stations in the Paris region (Massy, Marne-la-Vallée and Roissy-Charles-de-Gaulle airport), thereby providing a direct connection to the highspeed networks in the North, West and South-West of France without passing through Paris. The central station will be served by eight return TGV services which will take 45 min to travel from Rheims to the Gare de l'Est in Paris, as opposed to 1 hour $35 \mathrm{~min}$ currently with twelve return services, i.e. a $53 \%$ journey time reduction. The high-speed line will thus place Rheims at a similar time distance from the centre of Paris to towns in the second outermost concentric zone of the Paris region public transport network.

If the capacity of the conurbation of Rheims to become a regional metropolis depends on its ability to acquire the metropolitan attributes that have been described above, the arrival of the high-speed line can assist the process in several ways.

\subsection{Logistical attributes}

By improving accessibility, high-speed rail transport indubitably increases the concentration and redistribution of flows. If high-speed rail services are associated with good road, motorway and air accessibility, they encourage the centralization of activities which may assist the process of metropolization. In this context, the effects of high-speed rail depend on the quality of rail and road services in the area around the city which is served. 
The high-speed line does improve the accessibility of Rheims from, on the one hand, the centre of Paris and, on the other, the East of France, and more generally the provincial cities which are connected to the high-speed rail network, without passing through Paris. By increasing the accessibility of Roissy-Charles-de-Gaulle airport (30 min from the new station at Bezannes), it will connect the conurbation of Rheims to an international airport, which this nevertheless requires the provision of frequent services.

\subsection{Leadership attributes}

High-speed rail improves the accessibility of the area it serves from decision-making centres. It allows firms to canvass their clients more easily and vice-versa, and opens up local firms to competition from firms from outside, which may be a danger but also a factor for dynamism and innovation. It facilitates the existing connections with other centres (national and international) which are specialized in the development of new technologies, encourages the development of recognized centres of expertise and also facilitates the recruitment of executives and researchers.

The East-European high-speed line opens up the employment zones of Marne-la-Vallée and Roissy to Rheimsbased companies. However, in view of what occurred with other high-speed lines, the reduction in time-distance to the employment zones of the Paris region provided by the TGV did not systematically attract new populations (Bazin et al., 2006), nor increase long-distance commuting (INSEE-IAURIF, 2003). Only some conurbations with TGV services such as Tours, Nantes and Vendôme have seen an increase in their number of residents working in Paris.

Observations do not reveal a systematic increase in business trips either. The TGV-Atlantique was only responsible for a very large increase in trips taking less than half a day (a $260 \%$ increase). Its effects were also positive for some sectors, such as the study, consultancy, assistance sector and non-market services.

The effects of high-speed rail are therefore not only linked to the presence of the TGV but also to the economic situation in the city served, its distance from Paris, the pricing, the frequencies and the timetable, the local economic and social potential, and connections between the TGV and the local access network. High-speed rail is in this way a factor which reveals the strength and weaknesses of an area. It accentuates the attractiveness of areas which are already highly attractive, but can on the contrary hasten the decline of areas which are not.

The arrival of the East-European high-speed line could allow the conurbation of Rheims, which is located on the edge of the Paris region, to present itself as an alternative location. In order to do so, the area must be able to offer premises at attractive prices (or with attractive rents). With regard to offices, the Clairmarais business district which is being built behind Rheims central station, could increase the offer of services to firms. The high-speed line is also capable of assisting the development of the international or European dimensions of industrial activity with the improved access it provides to Roissy Airport. When it created Reims Champagne Développement three years ago, the conurbation of Rheims obtained the means of providing information about the competitive advantages of locating in Rheims with the arrival of the high-speed line. The local authorities and economic actors became aware of the need to envisage ways of accommodating new companies and there are a number of projects which increase supply in the medium term: in particular, the 90 hectare tertiary business park which is being built next to the new station at Bezannes. However, nothing is certain as although the actors are mobilized to provide land and business premises in the hope that firms will locate to the area because they are drawn by the high-speed line, the demand frequently comes from local firms which take advantage of fresh supply in order to increase the size of their premises or obtain premises that are more in line with present-day standards. Mixed outcomes in this regard have already been observed when other highspeed lines have been opened (Bazin et al., 2006).

\subsection{Identity attributes}

Not only does high-speed rail convey an image of modernity and progress to outside actors, it also improves the representation local actors and the population have of their area. The entry of Rheims into the group of cities with a TGV can help it to develop its European and international dimension, in particular by accentuating the international nature of business tourism and its congress centre. In addition, programmes to promote its cultural heritage and tourist potential are in progress, and should help to increase the city's influence. While these actions are not all directly linked to the high-speed line, it is this which was responsible for the realization that it was necessary to renew the city's image.

\subsection{The degree of coordination between actors}

The arrival of the high-speed line may be seen as a means of obtaining coordination between actors which therefore speeds up the realization of the need for it, as has already been observed in Lille (Paris, 2004). Moreover, some research dealing with a larger geographical area has shown that the benefits of high-speed rail depend on the extent to which the project receives collective support in the region (Ménérault, 1996, 1997 and 2000).

A consensus among the actors has appeared in Rheims and more largely between the conurbation and its environment, with regard for the need to use the arrival of the high-speed line in the near future to assist the area's development. This consensus has not yet resulted in genuine 
coordination which would recognize the indisputable role of Rheims as a driving force for the metropolization process. However, the future arrival of the high-speed line has already generated cooperation at four different geographical levels: between the conurbation, Épernay and Châlons-enChampagne, in the framework of the network of towns, in the département and, lastly, at regional level with regard to the reorganization of rapid regional rail services. A certain dynamic of thought and consultation has therefore developed around the advent of the TGV. It however needs to be strengthened by reinforcing the identity of each centre and its complementary relationship with Rheims and becoming institutionalized and concretized by projects.

\section{Conclusion}

The potential of Rheims for attraction is limited at national level, and very limited at international level, which at the present time prevents us from considering it to be a metropolis. The arrival in 2007 of the high-speed rail line will improve the accessibility of the areas it serves. It could also reinforce some metropolitan attributes by facilitating connections with other metropolises, facilitating the recruitment of executives and researchers and conveying an image of modernity. However, Rheims cannot take part in the metropolization race on its own, but must rely on the support of the urban areas around it. It must combat the fragmentation of policies and objectives which restrict its influence and orchestrate the efforts of interdependent areas which are linked by a common destiny and which will fail to achieve prosperity without it.

\section{Références}

Amiard, D., 1997. Le tourisme d'affaires et de congrès dans l'agglomération mancelle, In: Chevalier, J., Le Mans 6 ans après l'arrivée du TGV. Groupe de Recherche en Géographie Sociale, ESO, Espaces géographiques et Sociétés, Université du Maine, Le Mans, France.

AUDRR, 2004. Vers une métropolisation partagée. Rapport de l'Agence d'urbanisme et de développement de la région de Reims.

Ascher, F., 1995. Dynamiques métropolitaines et enjeux sociopolitiques. L'Harmattan, Paris.

Bazin, S., Beckerich, C., Delaplace, M., Masson, S., Petiot, R., 2004. La LGV : un outil d'ouverture des espaces et de renforcement de l'attractivité touristique ? Revue d'Économie méridionale, 52 (205-206), 57-83.

Bazin, S., Beckerich, C., Delaplace, M., Masson, S., 2006. Analyse prospective des impacts de la Ligne à grande vitesse Est-européenne dans l'agglomération rémoise et en région Champagne-Ardenne. Rapport final de recherche pour le Conseil Régional ChampagneArdenne, 2 tomes + annexes et synthèse.

Bellanger, F., 1991. Le TGV-Atlantique au Mans, à Saint-Pierre-des-Corps, à Tours et à Vendôme : opportunités, acteurs et enjeux. Rapport de la Maison des Sciences de la Ville, Université de Tours.

Brillaud, B., 2004. Vers l'émergence d'une métropole polynucléaire autour de Reims, In : AUDRR-INSEE Champagne-Ardenne, Les emplois métropolitains supérieurs, Reims... en quête de métropole. Rapport de l'Agence d'urbanisme et de développement de la région de Reims.

CESR, 2003. Métropolisation et maillage du territoire. Document de travail CESR Champagne-Ardenne, Châlons-en-Champagne.

Colletis-Wahl, K., Burmeister, A., 1996. TGV et fonctions tertiaires : grande vitesse et entreprises de service à Lille et à Valenciennes. Transports Urbains, 93.

Corade, N., 1994. La métropolisation ou la ville retrouvée ? Revue de la littérature actuelle. Revue d'Economie Régionale et Urbaine (RERU), 2, 251-266.

DATAR, 2003. Pour un rayonnement européen des métropoles françaises, Éléments de diagnostic et orientations. Paris, 48 p.

Derycke, P.-H., 1999. Comprendre les dynamiques métropolitaines, In : Lacour, C., Puissant, S. (eds), La métropolisation, croissance, diversité, fractures. Anthropos, collection Villes, Paris.

Facchinetti-Mannone, V., 2004. Les gares TGV périphériques : de nouveaux pôles de développement? In : Siino, C. (ed.), Métropolisation et grands équipements structurants. Presse Universitaire du Mirail, Toulouse, 305 p.

Gachelin, C., 1992. Métropolisation, dynamiques de métropolisation, Hypothèses pour la métropole Lilloise. Agence de développement et d'urbanisme de Lille.

Gilli, F., 2002. Le Bassin parisien, une région métropolitaine? Document de travail INSEE Picardie, $17 \mathrm{p}$.

Ginet, P., 2004. Processus de métropolisation à Anvers et à Liège : de l'élaboration du discours au renforcement de la cohérence territoriale métropolitaine, In : Métropoles en construction, Territoires, Politiques et Processus. L'Harmattan, Paris, 259-279.

IAURIF, 2002. Les zones d'activités économiques en Île-de-France, Note rapide sur le bilan du SDRIF. Rapport IAURIF, 309.

INSEE, 1999. Communes... mobilités, travail- études, CD ROM INSEE.

INSEE, 2002. Profils Nord-Pas-de-Calais, 2.

INSEE-IAURIF, 2003. Atlas des Franciliens, Tome 4 : activités et emplois. Rapport INSEE-IAURIF, Paris.

Joignaux, G., 1999. Euralille: Quelle métropolisation? Sciences de la Ville, 2, $14 \mathrm{p}$.

Klein, O., Claisse, G., 1997. Le TGV-Atlantique: entre récession et concurrence. Études et Recherches, Laboratoire d'économie des transports, Lyon.

Lacour, C., Puissant, S., 1999. La métropolisation, croissance, diversité, fractures. Anthropos, collection Villes, Paris, $190 \mathrm{p}$.

Menerault, P., 1996. Le TGV-Nord : appropriation locale d'un grand projet. Rapport INRETS, 96 p.

Menerault, P., 1997. Processus de territorialisation de la grande vitesse ferroviaire : le TGV et les régions, le cas du Nord-Pas-de-Calais. Rapport INRETS, $102 \mathrm{p}$.

Menerault, P., 2000. Appropriation régionale de la grande vitesse ferroviaire et rôle des régions dans la dynamique des réseaux : le cas du TGV en Wallonie et en Bretagne. Rapport GRRT.

Menerault, P, Stransky, V., 1999. La face cachée de l'intermodalité, Essai de représentation appliquée au couple TGV-air dans la desserte de Lille. les Cahiers Scientifiques du Transport, 35, 29-53.

Paris, D., 2004. Gouvernance des territoires, métropolisation et développement régional, Réflexions à partir de l'exemple de Lille et de sa région urbaine, In: Métropoles en construction, Territoires, Politiques et Processus. L'Harmattan, Paris, 21-38.

Reims champagne congrès expo, 2003. Bilan annuel. Reims.

Rozenblat, C., Cicille, P., 2003. Les villes européennes, Analyse comparative. La documentation française, Paris.

Scherr, T., 2004. L'impact du TGV-Est sur le tourisme d'affaires : quelles stratégies pour Reims et Epernay. Rapport de stage à la CCIRE, DESS de l'Université de Reims Champagne-Ardenne/ IATEUR.

Wackermann, G., 2000. Très grandes villes et métropolisation. Ellipses, Paris. 